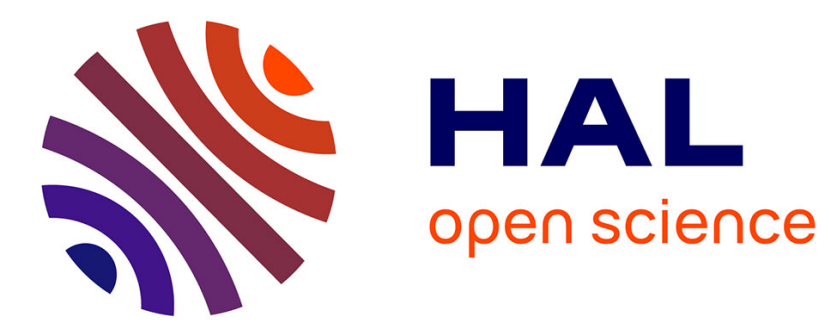

\title{
L'hybridation des instruments de gestion. L'exemple du pilotage de la performance universitaire en France
}

\author{
Laurent Mériade
}

\section{To cite this version:}

Laurent Mériade. L'hybridation des instruments de gestion. L'exemple du pilotage de la performance universitaire en France. Revue management \& avenir, 2019, Nº108 (2), pp.13. 10.3917/mav.108.0013 . hal-02163505

\section{HAL Id: hal-02163505 \\ https://hal.science/hal-02163505}

Submitted on 4 Dec 2019

HAL is a multi-disciplinary open access archive for the deposit and dissemination of scientific research documents, whether they are published or not. The documents may come from teaching and research institutions in France or abroad, or from public or private research centers.
L'archive ouverte pluridisciplinaire HAL, est destinée au dépôt et à la diffusion de documents scientifiques de niveau recherche, publiés ou non, émanant des établissements d'enseignement et de recherche français ou étrangers, des laboratoires publics ou privés. 


\title{
L'hybridation des instruments de gestion. L'exemple du pilotage de la performance universitaire en France
}

\begin{abstract}
Résumé
Si le mimétisme des instruments de gestion a été bien illustré notamment dans les organisations publiques, leur «hybridation » reste encore à définir conceptuellement. Dans cet article, nous proposons d'étudier comment les instruments de gestion s'articulent et se combinent entre eux.

Pour cela, à travers l'exemple des connexions entre le budget et les instruments de pilotage de la performance universitaire d'un $\mathrm{IUT}^{1}$, la théorie de la PET $^{2}$ nous permet de circonscrire les premiers contours d'un processus d'hybridation instrumentale.
\end{abstract}

Mots clés. budget, performance, instruments de gestion, équilibre ponctué, hybridation

\section{The hybridization of management instruments. The example of university performance management in France}

\begin{abstract}
While the mimicry of management instruments has been well illustrated, particularly in public organizations, their "hybridization" has yet to be defined. In this article, we propose to study how management instruments are linked together.

For this, through the example of the connections between the budget and the performance management instruments of an IUT, the theory of PET allows us to circumscribe the first outlines of an instrumental hybridization process.
\end{abstract}

Keywords. budget, performance, management instruments, punctuated balance, hybridization

\footnotetext{
${ }^{1}$ Institut Universitaire de Technologie
}

${ }^{2}$ Ponctuated Equilibrium Theory 


\section{Introduction}

« Mimétisme » et «hybridation » caractérisent aujourd'hui le déploiement des instruments de gestion dans les organisations. Ceci est particulièrement manifeste dans les organisations publiques où se déploient et se rencontrent des outils empruntés à la fois au monde marchand (comptabilité analytique, tableaux de bord) et au management public de l'État (budget, contrats d'objectifs et de moyens) (Dupuis, 2015).

Si le mimétisme instrumental a été particulièrement bien illustré (Trosa, 1989; Kieffer, 1996), l'hybridation des instruments, plus récente, notamment dans les organisations publiques, reste encore à définir conceptuellement (Émery, 2010). A travers l'exemple de la gestion des qualifications et des compétences, Oiry (2005) a suggéré l'existence d'un processus d'hybridation des instruments de gestion et il incite les chercheurs à se focaliser sur sa description détaillée.

L'instrument de gestion est défini à la fois comme une technique et un usage ce qui le distingue de l'outil principalement étudié dans ses dimensions techniques (Bourguignon 2006). Son hybridation peut s'opérer à la fois par ses modalités d'utilisation mais également par l'intermédiaire de son substrat technique (Hatchuel et Weil, 1992). Cette hybridation a essentiellement été illustrée à travers l'étude des pratiques ou des valeurs et logiques portées par ces instruments (de Vaujany et Grimand, 2005 ; de Vaujany, 2006, Emery et Giauque, 2014, Buffat, 2014). Par contre, son approche technique, qualifiée ici d'instrumentale, est très peu analysée.

La problématique de recherche abordée dans cet article est donc la suivante : Comment les instruments de gestion s'articulent ou se combinent à partir de leurs caractéristiques techniques ?

Pour aborder cette question nous privilégions une approche instrumentale et technique des instruments de gestion (Rabardel, 2002) favorisant une analyse fine de ces instruments.

L'hybridation a été d'abord définie dans les travaux des sciences de la nature pour caractériser la combinaison d'une espèce A avec une autre B (Amslem, 2013). Nous devons l'apparition du concept d'hybridation des instruments de gestion aux économistes et notamment à ceux de la Régulation en France. Abo (1994) parle le premier d'hybridation des instruments pour décrire les situations de fusion de plusieurs modèles de gestion à travers l'utilisation de plusieurs instruments issus de ces modèles. Pour Boyer (1998), l'hybridation se définit comme la rencontre de deux forces incarnées par des instruments et débouchant sur un nouveau modèle de management. Assez paradoxalement en sciences de gestion, le processus de combinaison d'instruments de gestion n'a été souligné que par les travaux sur l'hybridation instrumentale (Meyer et Hammerschmid, 2006 ; Meyer et al., 2014 ; Oiry, op.cit.) sans en faire, pour autant, une description exhaustive.

Les universités françaises constituent un champ d'étude exemplaire de cette hybridation des instruments de gestion dans lequel les procédures budgétaires traditionnelles se sont vues progressivement associer un outillage de mesure et de pilotage de la performance particulièrement abondant. Par contre, si les changements législatifs ou institutionnels de ses dix dernières années ( $\mathrm{LRU}^{3}, \mathrm{RCE}^{4}$, loi Fioraso5) ont largement développé cet outillage, ils n'ont pas nécessairement pensé ses articulations avec les instruments de gestion existants. Or, nous constatons que dans les pratiques universitaires, les décisions de gestion continuent à être essentiellement formulées lors de la préparation des $\mathrm{DOB}^{6}$ et des dialogues budgétaires avec les composantes.

En effet, en matière de gestion publique, le budget a toujours joué un rôle-clé (Parker, 2011 ; Arnaboldi et $a l ., 2015)$ car il est souvent le principal support de négociation des objectifs et moyens attribués à une organisation publique. Aussi, il parait opportun d'étudier ses capacités à participer au pilotage de la performance dans l'univers hybride des modèles post-bureaucratiques (Emery, 2012) orientées à la fois vers la gestion des moyens et des résultats.

Dès lors, l'objectif de notre recherche vise à analyser les articulations qui peuvent exister entre ces décisions budgétaires et les instruments de pilotage de la performance universitaire.

Pour cela, en matière d'analyse instrumentale des budgets publics, deux conceptions à la fois complémentaires et opposées cohabitent. D'un côté, celle de l'incrémentalisme budgétaire (Wildavsky,

\footnotetext{
3 Libertés et Responsabilités des Universités en 2007

4 Responsabilités et Compétences Elargies à partir du $1^{\text {er }}$ janvier 2009

5 Juillet 2013

${ }^{6}$ Débats d'Orientation Budgétaires
} 
1964 ; Berry, 1990) qui considère que les choix budgétaires sont relativement peu contraints par l'univers dans lequel ils sont mobilisés, ce qui rend leurs évolutions relativement linéaires. D'un autre côté, la conception de l'équilibre ponctué (Baumgartner et Jones, 1993) considère que l'incrémentalisme n'est pas permanent. Il est ponctué par des changements intervenants dans l'univers des organisations publiques que les décideurs intègrent dans leurs décisions budgétaires à travers des ponctuations très marquées (Baumgartner et al., 2009). Ces ponctuations expriment l'adaptation des budgets aux changements intervenants dans leurs environnements. Au départ développée pour analyser les changements organisationnels (Romanelli et Tushman, 1994), puis appliquée par la suite à l'étude des budgets publics, l'approche de la $\mathrm{PET}^{7}$ a progressivement permis d'éclairer de manière très approfondie les relations entre le budget et les évolutions de son environnement institutionnel et organisationnel (Eissler et al., 2016). Nous testerons son aptitude à décrire les éventuels liens pouvant exister entre le budget et les instruments de pilotage de la performance des universités françaises.

Pour cela, notre travail s'organise en trois temps. Après avoir analysé la littérature en matière d'hybridation nous analysons le cas longitudinal d'un IUT $^{8}$ français à travers le modèle de la PET. Nous constatons que le budget s'hybride avec les indicateurs de performance universitaires prescrits et nous démontrons que cette hybridation instrumentale (Meyer et Hammerschmid, 2006) se réalise au moins à trois niveaux (stratégique, organisationnel et artéfactuel) correspondant aux trois principales dimensions d'un outil de gestion: sa philosophie gestionnaire, sa vision simplifiée de l'organisation et son substrat technique (Hatchuel et Weil, 1992).

\section{Revue de littérature}

La littérature envisage deux hypothèses contradictoires quant à la manière dont les instruments de gestion peuvent évoluer les uns par rapport aux autres (Sandelin, 2008). La première suggère l'existence de complémentarités entre ces outils. De nombreux auteurs ont développé cette approche en considérant le contrôle de gestion comme un «package » (Ferreira et Otley, 2009) ou un «système » (Malmi et Brown, 2008) de pilotage coordonné et intégré. La seconde, à l'inverse, repose sur l'idée que la reconfiguration du système de contrôle s'opère via des mécanismes de substitution et de concurrence entre les instruments de gestion (Banker et al. 2000; Hoque, 2005). Il parait particulièrement intéressant de positionner les articulations entre budget et instruments de mesure de la performance universitaire sur cette échelle qui va de la complémentarité à la concurrence entre les instruments de gestion. En effet, la manière dont ces instruments de gestion cohabitent constitue une caractéristique très visible de l'univers hybride des administrations publiques (Emery et Giauque, 2014) dont l'illustration dans la littérature reste, paradoxalement, assez peu développée.

Le concept d'hybridité fait référence à la coexistence d'éléments contradictoires (Denis et al., 2015). Cette hybridité peut se construire à partir de diverses contradictions qui vont de la dialectique au paradoxe en passant par le dilemme (Smith et Lewis, 2011). Alors que dans la dialectique les éléments contradictoires sont réunis et intégrés dans une solution, dans le dilemme, un choix doit être effectué entre ces éléments. Dans le paradoxe, aucun choix entre ces éléments n'est effectué (ibid.). L'hybridité constitue le résultat de l'ensemble de ces choix et non choix qui s'exprimera sous diverses formes (intégration, suppression, cohabitation des éléments contradictoires).

Plus particulièrement, dans le secteur public, l'hybridité correspond à une combinaison de logiques de gestion et d'organisation souvent antinomiques (par exemple ceux du NPM et de la bureaucratie weberienne) qui génèrent des rôles et des pratiques hybrides (Denis et al., op.cit.). Une part importante des recherches s'est concentrée sur l'hybridité des gouvernances (publique et privée) et des organisations (Da et al., 2018). D'autres travaux s'intéressent à l'hybridité des pratiques et l'illustrent par leurs effets sur les comportements des acteurs (Teelken, 2015). Par contre, nous notons une absence de travaux sur les effets de cette hybridité sur les instruments de gestion pourtant souvent à l'origine de ces comportements ou pratiques hybrides.

Dans le secteur public français, et plus particulièrement dans les organisations universitaires, cette hybridité s'exprime de manière très explicite souvent sous l'influence de l'introduction et l'exploitation de multiples instruments de gestion issus à la fois des principes de management public et privé. Pour mieux

\footnotetext{
${ }^{7}$ Ponctuated Equilibrium Theory

${ }^{8}$ Institut Universitaire de Technologie
} 
comprendre cette hybridité (que nous qualifions ici d'hybridation instrumentale), il devient dès lors très pertinent d'étudier comment ses instruments s'articulent entre eux soit pour prendre de nouvelles formes soit pour renforcer leurs formes précédentes. Le cas, dans les universités françaises, de la cohabitation récente entre les instruments de pilotage de la performance et les budgets des établissements constituent un champ d'étude particulièrement adapté à l'analyse en profondeur de l'hybridation instrumentale.

En France, à partir du début des années 2000, les universités se sont vues prescrire une obligation de performance financière (Mignot-Girard, 2012) à partir d'un ensemble d'indicateurs de performance d'abord définis à travers les PAP $^{9}$ des programmes 150 (Formation et recherche universitaire) et 231 (Vie étudiante) de la MIRES ${ }^{10}$.

Ces changements inspirés notamment par La LOLF ${ }^{11}$ avaient pour ambition de relier les décisions budgétaires avec des indicateurs de performance représentatifs des principales dépenses de l'État (Dreveton et al. 2012). Dans les universités françaises, la LRU et le passage aux RCE ont, par la suite, accompagné ces évolutions en accordant une plus grande autonomie de gestion aux directions universitaires dans le choix de leurs instruments de gestion (Boitier et Rivière, 2013).

Au sein des systèmes de contrôle des universités françaises, le budget demeure dominant à la fois car il trace les flux financiers mais aussi car il demeure le langage utilisé dans le pilotage de la performance et l'allocation des moyens (Boitier et Rivière, 2016). D'une part, les budgets sont utilisés pour développer dans les universités l'usage et la prise en compte d'indicateurs de performance définis et prescrits de façon globale (ibid.). D'autre part, Moll et Hoque (2011) soulignent que les acteurs internes de l'université par leurs interventions sur les décisions budgétaires cherchent à faire avancer leur propre vision de la performance. L'adoption des instruments de pilotage de la performance universitaire ne se réalise donc pas de manière mimétique et linéaire mais au contraire à travers des interactions complexes (Ezzamel et al. 2012). Si les universités, et surtout leurs composantes, se soumettent aux processus d'évaluation de la performance, elles contournent souvent la mise en œuvre en interne du financement conditionnel liées cette évaluation (Boitier et Rivière, 2016, op.cit.) au profit du maintien de procédures budgétaires traditionnelles. Pour Wyser (2010), il apparait ainsi que les nouvelles logiques de gestion publique inspirées du NPM ${ }^{12}$ sont venues s'ajouter à des modalités de gestion plus traditionnelles de gestion sans réellement questionner leurs articulations les unes par rapport aux autres.

Pour Emery et Giauque (2014), si le constat de l'hybridation est désormais conventionnel en management public (Rondeaux, 2014 ; Buffat, 2014), la question des formes et modalités que peut revêtir l'articulation des logiques publique et privée reste à penser. En effet, les principaux travaux ont surtout insisté sur l'addition de ces logiques en mettant en avant leurs effets sur l'organisation publique (ibid.), les valeurs ou encore les statuts publics (Emery et Giauque, 2014). D'autres travaux ont cherché à analyser les points de conjonction et d'articulation entre ces logiques en étudiant les perceptions des usagers (Guenoun et al., 2016) ou celle des agents publics (Kletz et al., 2014; Mazouz et al, 2015). Bien conscient que l'introduction dans le secteur public de nouveaux instruments de gestion issus du secteur privé reste une manifestation essentielle de ces articulations de logiques, Meyer et Hammerschmid (2006) ont introduit le concept d'hybridation instrumentale. Ils l'étudient principalement en vue de circonscrire les multiples effets que cette hybridation peut générer sur l'identité et le comportement des acteurs (Meyer et al., 2014). En complément de ces travaux, il est important d'analyser, à travers l'évolution des instruments, comment, de manière pratique, cette hybridation instrumentale s'effectue. En effet, les instruments de gestion représentent des objets privilégiés de rencontre mais aussi de tensions entre les logiques de gestion qui traversent les organisations publiques. Le concept d'instrument est défini par l'association d'un substrat (outil) technique et d'un schème d'action (Lorino, 2005). Les travaux sur l'appropriation des outils de gestion (De Vaujany, 2006; De Vaujany et Grimand, 2005) décrivent, de manière microanalytique, la manière dont les schèmes d'action se construisent et viennent s'adosser à l'outil de gestion. En s'intéressant aux interactions entre l'outil et ses utilisateurs ils peuvent contribuer à une meilleure compréhension de l'hybridation instrumentale qui se focalise plus spécifiquement sur les

\footnotetext{
${ }^{9}$ Projet Annuel de Performance

10 Mission Interministérielle pour la Recherche et l'Enseignement Supérieur

${ }^{11}$ Loi Organique sur les Lois de Finances

${ }^{12}$ New Public Management
} 
interactions entre les instruments.

A ce titre dans ces organisations, les budgets occupent une place centrale qui les connectent avec les autres instruments de gestion. En effet, les budgets ont la faculté d'autoriser un couplage lâche (loose coupling, Modell, 2003) entre les objectifs de performance prescrits et les intérêts et rationalités des principaux acteurs universitaires (directeurs de composantes, enseignants, responsables administratifs et financiers, personnels administratifs et techniques, étudiants). Ce couplage lâche constitue une incitation à la construction de décisions budgétaires prenant en compte des intérêts locaux mais s'éloignant parfois, de manière significative, des cibles de performance prescrites (Bollecker, 2013). Parce qu'il est à la fois un système « ouvert et fermé » mais aussi « spontané et intentionnel » (Orton et Weick, 1990, p. 204205), le budget dispose d'un niveau de perméabilité important vis-à-vis des autres instruments de gestion de universités. Dès lors, il permet de restituer une grande part du processus d'hybridation dans la mesure où il demeure l'instrument de gestion majoritairement utilisé par les parties prenantes. Ce couplage faible explique en partie la place persistante qu'occupe les budgets dans la plupart des organisations dans lesquelles les velléités de gestion sans budget n'ont pas profondément modifié les pratiques budgétaires (Libby et Lindsay, 2010).

Pour Orlikowski (2006, p. 462), le budget constitue un «échafaudage » qui se construit dans la pratique et qui façonne les interactions et les activités. Le budget perçu comme échafaudage de l'action (ibid.) se caractérise par sa flexibilité et sa capacité à transformer l'outillage de gestion des organisations. La théorie de l'équilibre ponctué (Baumgartner et al., 2009) offre l'opportunité d'analyser les budgets des organisations publiques et leurs éventuelles relations avec l'outillage de pilotage de la performance.

\section{Cadre conceptuel}

Les principaux travaux analysant les processus de construction des budgets ont principalement justifié les choix budgétaires soit par des raisons idéologiques ou partisanes (Franzese 2002 ; Blais et al, 2010) soit par la lourdeur des organisations et l'information limitée de ses décideurs (Wildavsky,1964). En se focalisant sur de longues périodes (5 à 10 ans voire plus), ces analyses font principalement apparaitre des variations limitées des budgets qui justifient, dans le temps, un important «incrémentalisme budgétaire » consistant à moduler de manière marginale les budgets des années précédentes : "largest determining factor of the size and content of this year's budget is last year's budget " (Wildavsky,1964, p. 13). Si ces approches disposent d'une importante robustesse pour des analyses longitudinales, elles ne permettent pas d'expliquer des variations budgétaires qui interviennent de manière ponctuelle au cours de courtes périodes.

Le modèle de la PET (Baumgartner et Jones, 1993) s'est construit afin de mieux comprendre ces évolutions de courtes périodes. Il reprend une partie des conclusions de la «théorie de l'équilibre ponctué de changements radicaux»(Romanelli et Tushman, 1994) qui explique les évolutions des organisations par de longues périodes de relative stabilité entrecoupées de courtes périodes de changements majeurs et discontinus appelés transformations (ibid.). En l'appliquant ces résultats à l'analyse des budgets publics, Baumgartner et Jones (1993) montrent que les distributions budgétaires se particularisent également par de longues périodes de décisions incrémentales interrompues par un pic central, appelé ponctuation.

Ils justifient ces ponctuations par l'apparition de frictions institutionnelles et cognitives qu'ils définissent comme des changements de problèmes et variables surveillés par les décideurs publics qui, en rentrant en contact avec les budgets, imposent de forts ajustements. Baumgartner et Jones (1993) illustrent ce phénomène à partir de l'exemple du budget fédéral des Etats Unis, dans les année 1970, pour lequel des variables jusqu'alors non surveillées (le niveau de pollution des villes ou la consommation de tabac) vont faire l'objet d'une attention particulière et nécessiter des plans de prévention exceptionnels provoquant de fortes ponctuations budgétaires.

Pour ces auteurs, les frictions institutionnelles proviennent de choix de procédures (normes ou règles administratives) qui, en privilégiant la gestion de problèmes spécifiques, vont constituer des résistances internes. Celles-ci empêchent les réformes des institutions et renforcent les politiques de statu quo budgétaires. Aussi, lorsque des changements de procédures interviennent, elles imposent de la part des décideurs de forts ajustements budgétaires afin d'adapter les budgets à ces évolutions (Jones et Baumgartner, 2012). 
Les frictions cognitives s'expliquent par le fait que les décideurs budgétaires, ne pouvant pas surveiller simultanément chacune des milliers de variables qui impactent leurs budgets, ne prêtent attention qu'à un nombre limité de variables jugées essentielles. Lorsque les effets de certaines variables non surveillées apparaissent, ils doivent pratiquer des ajustements budgétaires exceptionnels (Baumgartner et al, 2009).

Le cadre d'analyse de Baumgartner et al. (2009) a fait l'objet, dans un premier temps, d'une mobilisation pour analyser des choix budgétaires nationaux, mais il est aujourd'hui utilisé, de manière assez robuste, à un niveau intermédiaire de prise de décision telles que le prouvent des études récentes sur les ponctuations budgétaires dans les collectivités locales ou des services déconcentrés de l'Etat (Baumgartner et al, 2011). Dans une analyse récente de leur cadre théorique, Jones et Baumgartner (2012) insistent sur l'impératif d'approfondissement de la PET à partir d'études de terrain pour évaluer les processus réels de décision.

Nous cherchons à répondre à cette attente, en testant ce cadre d'analyse, à travers de l'étude de cas unique d'un établissement universitaire, afin de mieux saisir le processus de choix budgétaires à un niveau local.

\section{Méthodologie}

Notre méthodologie repose sur une approche instrumentale qui vise à étudier les outils de gestion dans un même mouvement dialectique quelle que soit leur nature matérielle (Rabardel, 2002, p.272). Cette approche préconise une étude engagée dans l'action pour laquelle l'étude de cas est particulièrement adaptée (Derujinsky-Laguecir et al., 2011). Elle peut notamment permettre de rendre compte des écarts entre les utilisations effectives des instruments de gestion et les prescriptions des concepteurs (ibid.).

Pour mener cette démarche, nous revisitons les modalités d'une participation observante (Peretz, 2004) effectuée dans le cadre d'une recherche action (David et al., 2000), au sein d'un IUT de plus de 3000 étudiants créé en 1969. Par leur statut jusqu'alors dérogatoire (article 713.9, ancien article 33), les IUT se trouvent directement impactés sur le plan budgétaire par le caractère mouvant des outils de pilotage de la performance prescrits par leurs universités et leurs instances ministérielles de tutelle (DGESIP ${ }^{13}$, AERES $^{14}$ puis HCERES $^{15}$ ). Jusqu'en 2007, bien des aspects budgétaires des IUT dépendaient d'une régulation directe avec le Ministère de l'enseignement supérieur. Avec la LRU, la définition de l'offre de formation et du budget ainsi que la gestion des ressources humaines sont négociés au niveau local avec les présidences d'université (Agulhon, 2018). Ceci a créé dans les IUT un changement radical de pratiques de gestion et de capacités à négocier. Chargé de mission «Finances et budget » au sein de cet IUT, nous avons participé, au cours des années étudiées (2011 à 2015), aux dialogues budgétaires avec l'Université, aux évaluations ministérielles et aux divers conseils de gestion de cet établissement. Le choix de cette période d'étude a été définie ainsi car, d'une part, elle coïncide, pour cette université, au passage aux Responsabilités et Compétences Elargies (2011) qui correspond à un développement sensible des outils de pilotage interne de cet établissement et de ses composantes. D'autre part, cette période d'étude permettait à l'auteur, durant sa fonction de chargé de mission, d'accéder à des informations très spécifiques, recensées dans un carnet de bord personnel, auxquelles il nous aurait été plus difficile d'accéder dans d'autres établissements ou départements universitaires. Néanmoins cette forte implication porte en elle un biais d'objectivité évident qui impose de la part du chercheur des allersretours très réguliers entre appartenance et distanciation (Lalonde, 2013).

Dans l'observation participante la priorité est à la distanciation du chercheur afin de réduire la part de subjectivité qui pourrait provenir d'une implication trop importante (ibid.). Au contraire, la participation observante permet d'accepter cette subjectivité en incitant le chercheur à s'affirmer en tant que membre à part entière de son terrain de recherche (Lalonde, 2013). En sciences de gestion, cette subjectivité peut parfois être considérée comme un avantage en la reconnaissant au lieu de la bloquer (ibid.).

La priorité accordée à la participation peut parfois constituer la condition sine qua non de la réalisation de l'observation (Soulé, 2007). Dans le cadre de notre travail, l'implication de l'auteur est consubstantielle à l'activité étudiée. Ici, elle permet de se rapprocher sans retenue d'objets de recherche techniques (le budget ou les tableaux de bord) dont l'analyse nécessite un niveau de compréhension étendue des mécanismes de construction afin, ensuite, de saisir au mieux leurs

\footnotetext{
13 Direction Générale pour l'enseignement supérieur et l'insertion professionnelle

14 Agence d'évaluation de la recherche et de l'enseignement supérieur

15 Haut Conseil de l'évaluation de la recherche et de l'enseignement supérieur
} 
interactions. Il n'en reste pas moins que cette forte implication porte en elle un biais d'objectivité évident qui impose, de la part du chercheur, des allers-retours très réguliers entre appartenance et distanciation (Lalonde, op.cit.). Dans ce travail, ces allers-retours ont pu se réaliser avec plus d'aisance dans la mesure où le chercheur, s'il est l'architecte des outils, n'en est pas le décideur. Ceci le rend très peu responsable des choix de gestion budgétaire qui sont au centre de cette étude.

Pour réaliser cette analyse, nos données empiriques sont de deux ordres. Premièrement, des données budgétaires issues des documents et décisions budgétaires de 2011 à 2015 de cet IUT que nous analysons à partir d'une analyse longitudinale des principaux chapitres budgétaires afin de rechercher les ponctuations significatives intervenues au cours de cette période. Deuxièmement, des données issues des indicateurs prescrits au niveau ministériel (MIRES et DGESIP) et construit au niveau local (tableaux de bord de gestion) nous permettent d'appliquer le modèle de la PET en recherchant les frictions institutionnelles et cognitives que ces indicateurs peuvent provoquer.

\section{Trois niveaux d'instrumentation de la performance dans les universités françaises}

Dans le cadre de notre étude de cas, les ajustements budgétaires majeurs que nous constatons peuvent être associés à un recentrage progressif de l'attention accordée à la performance universitaire autour d'objectifs ou d'indicateurs prescrits au niveau central ou construits au niveau local. Il est possible de distinguer au moins trois niveaux d'objectifs et indicateurs de performance universitaire. D'une part, les niveaux législatif et ministériel représentant le contexte institutionnel de notre cadre conceptuel et, d'autre part, le niveau local représentant le contexte cognitif d'adaptation des indicateurs prescrits aux niveaux supérieurs.

\subsection{Le niveau législatif}

Ce premier niveau se structure autour des indicateurs issus des objectifs et indicateurs votés annuellement dans le $\mathrm{PLF}^{16}$ et se rapportant au programme 150 «Formations supérieures et recherche universitaire », principal programme géré par le Ministère de l'enseignement supérieur. Une analyse longitudinale de ce programme révèle une «mouvance » significative des objectifs et indicateurs que l'on peut apparenter à des frictions institutionnelles au sens de Baumgartner et al (2009). En effet, entre les PAP ${ }^{17} 2011$ (tableau 1) et 2015 (tableau 2), nous constatons la suppression de trois objectifs de performance (Objectif $n^{\circ} 7$ Produire des connaissances scientifiques au meilleur niveau international; Objectif $n^{\circ} 8$ Améliorer le transfert et la valorisation des résultats de la recherche; Objectif $\mathrm{n}^{\circ} 9$ Consolider l'Europe de la recherche). Ceci constitue une première forme de changements institutionnels qui au contact du budget de l'établissement étudié provoquent de fortes ponctuations.

\footnotetext{
${ }^{16}$ Projet de Loi de Finances

${ }^{17}$ Projet Annuel de Performance
} 
Tableau 1. Indicateurs PAP Programme 150 « Formations supérieures et recherche universitaire » année 2011 (Source : MESR $^{18}$ )

\begin{tabular}{|c|}
\hline Indicateurs \\
\hline $\begin{array}{l}\text { OBJECTIF } 1 \text { Répondre aux besoins de qualification supérieure par la formation initiale } \\
\text { et continue } \\
\text { - INDICATEUR } 1.1 \text { Pourcentage d'une classe d'âge titulaire d'un diplôme de l'enseignement } \\
\text { supérieur en formation initiale } \\
\text { - INDICATEUR } 1.2 \text { Insertion professionnelle des jeunes diplômés } \\
\text { - INDICATEUR } 1.3 \text { Formation tout au long de la vie }\end{array}$ \\
\hline $\begin{array}{l}\text { OBJECTIF } 2 \text { Améliorer la réussite des étudiants } \\
\text { - INDICATEUR 2.1 Jeunes sortant de l'enseignement supérieur sans diplôme post-bac } \\
\text { - INDICATEUR 2.2 Réussite au DUT et BTS } \\
\text { - INDICATEUR 2.3 Réussite en L } \\
\text { - INDICATEUR 2.4 Réussite en M } \\
\text { - INDICATEUR 2.5 Réussite en D }\end{array}$ \\
\hline
\end{tabular}

OBJECTIF 3 Faire de l'enseignement supérieur un outil performant de formation tout au long de la vie

- INDICATEUR 3.1 Pourcentage de diplômés en formation continue par rapport au nombre total de diplômés

- INDICATEUR 3.2 Nombre des validations des acquis de l'expérience

OBJECTIF 4 Accroître l'attractivité internationale

- INDICATEUR 4.1 Part des étudiants étrangers inscrits en master et doctorat

- INDICATEUR 4.2 Nouveaux inscrits pour une thèse en co-tutelle

- INDICATEUR 4.3 Proportion d'étrangers dans les recrutements d'enseignementschercheurs

OBJECTIF 5 Optimiser l'accès aux ressources documentaires pour la formation et la recherche

- INDICATEUR 5.1 Mesure de la disponibilité hebdomadaire des places de bibliothèques

- INDICATEUR 5.2 Fréquentation des bibliothèques par lecteur inscrit

OBJECTIF 6. Optimiser l'offre de formation et la gestion des établissements de l'enseignement supérieur

- INDICATEUR 6.1 Part des mentions à faible effectif ( $L$ et $M)$

- INDICATEUR 6.2 Part des ressources propres liées à des prestations de service sur les recettes totales des établissements

- INDICATEUR 6.3 Pourcentage de sites secondaires dont le nombre d'étudiants est inférieur à 1000

- INDICATEUR 6.4 Taux d'occupation des locaux

OBJECTIF 7 Produire des connaissances scientifiques au meilleur niveau international et accroître le dynamisme des équipes de recherche

- INDICATEUR 7.1 Production scientifique des opérateurs du programme

- INDICATEUR 7.2 Reconnaissance scientifique des opérateurs du programme

- INDICATEUR 7.3 Proportion des enseignants-chercheurs produisants

OBJECTIF 8 Améliorer le transfert et la valorisation des résultats de la recherche

- INDICATEUR 8.1 Part des ressources apportées aux opérateurs par les redevances sur titres de propriété intellectuelle

- INDICATEUR 8.2 Part des contrats de recherche passés avec les entreprises dans les ressources des opérateurs

OBJECTIF 9 Consolider l'Europe de la recherche

- INDICATEUR 9.1Taux de présence des opérateurs du programme dans les projets financés par le PCRD de l'Union européenne

- INDICATEUR 9.2 Part des articles co-publiés avec un pays membre de l'Union européenne (UE 27) dans les articles des opérateurs du programme

\footnotetext{
${ }^{18}$ Ministère de l'Enseignement Supérieur et de la Recherche
} 
Tableau 2. Indicateurs PAP Programme 150 «Formations supérieures et recherche universitaire » année 2015 (Source : MESR)

\begin{tabular}{|c|}
\hline Indicateurs \\
\hline $\begin{array}{l}\text { OBJECTIF } 1 \text { Répondre aux besoins de qualification supérieure par la formation tout au } \\
\text { long de la vie } \\
\text { INDICATEUR } 1.1 \text { Pourcentage d'une classe d'âge obtenant un diplôme de l'enseignement } \\
\text { supérieur en formation initiale } \\
\text { INDICATEUR } 1.2 \text { Insertion professionnelle des diplômés en formation initiale } \\
\text { INDICATEUR } 1.3 \text { Formation continue }\end{array}$ \\
\hline $\begin{array}{l}\text { OBJECTIF } 2 \text { Améliorer la réussite des étudiants } \\
\text { INDICATEUR 2.1 Jeunes sortant de l'enseignement supérieur sans diplôme post-bac } \\
\text { INDICATEUR 2.2 Réussite à l'examen de BTS } \\
\text { INDICATEUR 2.3 Réussite en 1er cycle universitaire } \\
\text { INDICATEUR 2.4 Réussite en M } \\
\text { INDICATEUR 2.5 Réussite en D }\end{array}$ \\
\hline $\begin{array}{l}\text { OBJECTIF } 3 \text { Produire des connaissances scientifiques au meilleur niveau intern } \\
\text { INDICATEUR } 3.1 \text { Production scientifique des opérateurs du programme }\end{array}$ \\
\hline $\begin{array}{l}\text { OBJECTIF } 4 \text { Améliorer le transfert et la valorisation des résultats de la recherche } \\
\text { INDICATEUR } 4.1 \text { Montant des redevances sur titre de propriété intellectuelle dans les } \\
\text { ressources des opérateurs } \\
\text { INDICATEUR } 4.2 \text { Montant des contrats de recherche passés avec les entreprises dans les } \\
\text { ressources des opérateurs }\end{array}$ \\
\hline $\begin{array}{l}\text { OBJECTIF 5 Renforcer l'ouverture européenne et internationale des établissements } \\
\text { INDICATEUR 5.1 Part des étudiants étrangers inscrits en Master et en Doctorat } \\
\text { INDICATEUR 5.2 Proportion d'étrangers dans les recrutements d'enseignants-chercheurs } \\
\text { INDICATEUR 5.3 Coopération internationale } \\
\text { INDICATEUR 5.4 Taux de présence des opérateurs du programme dans les projets financés } \\
\text { par le PCRD de l'Union Européenne }\end{array}$ \\
\hline $\begin{array}{l}\text { OBJECTIF } 6 \text { Améliorer l'efficience des opérateurs } \\
\text { INDICATEUR 6.1 Part des mentions à faibles effectifs ( } L \text { et } M \text { ) } \\
\text { INDICATEUR 6.2 Mesure de la disponibilité hebdomadaire des places de bibliothèque }\end{array}$ \\
\hline
\end{tabular}

En effet, la suppression de ces objectifs impacte les choix budgétaires des universités constatables dans des ponctuations apparaissant à la fois au niveau des unités budgétaires (UB) et des budgets des départements de formation et recherche. Dans notre étude de cas, ces ponctuations se concrétisent, tout d'abord, à partir de 2015, par une forte baisse des dépenses de recherche (graphique 1).

Graphique 1. Evolution dépenses de recherche 2011-2015 (en $€$ )

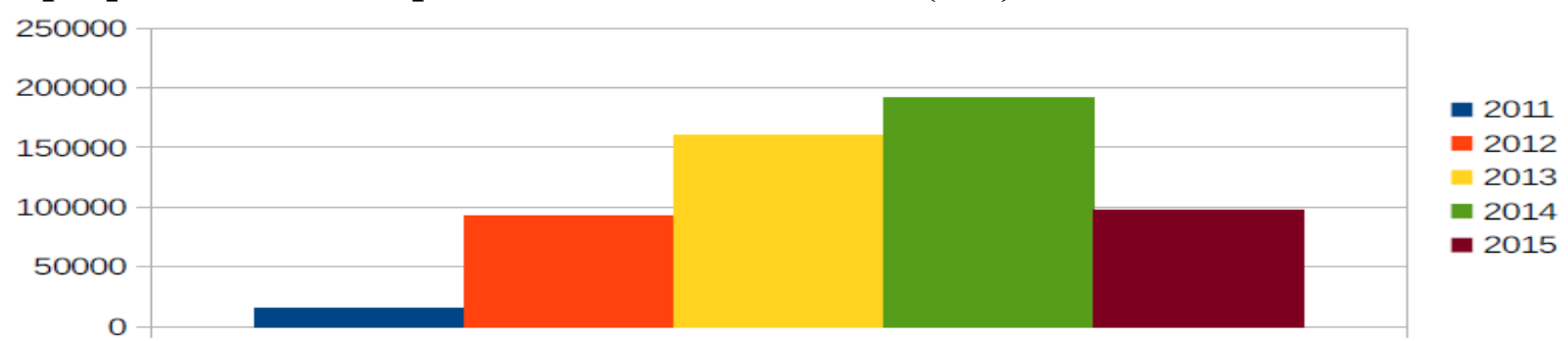

Par ailleurs, l'analyse comparative des PAP 2011 et 2015 révèle également la suppression de certains indicateurs relatifs au nombre de $\operatorname{VAE}^{19}$ (indicateur 3.2. tableau 1) et au nombre de sites universitaires de petite taille (indicateur 6.3. tableau 1). Ceci représente également des frictions institutionnelles dans la mesure où ces changements génèrent, à partir de 2015, afin de prendre en charge la disparition des limitations de crédits liées à ces deux indicateurs, un ajustement budgétaire à la hausse des dotations de fonctionnement accordées par l'Université à cet IUT (graphique 2)

\footnotetext{
${ }^{19}$ Validation des Acquis de l'Expérience
} 


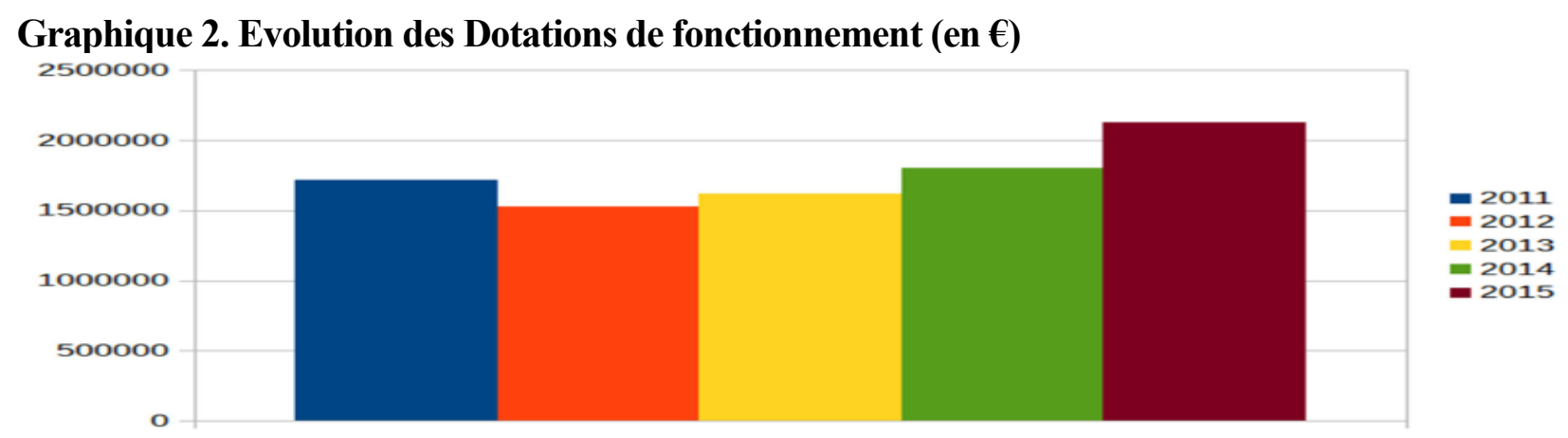

Cette analyse des PAP met également en lumière l'apparition en 2015 de nouveaux indicateurs de performance (tableau 2) portant notamment sur de nouveaux objectifs en matière d'insertion professionnelle (indicateur 1.2.), de réussite des étudiants (indicateur 2.3.) et de coopération internationale (indicateur 5.3.). Ceci crée autant de nouvelles frictions institutionnelles imposant, en 2015, des ponctuations budgétaires pour ajuster plus nettement à la baisse les effectifs d'étudiants (graphique 3) et à la hausse le budget des recettes des relations internationales (graphique 4).

Graphique 3. Evolution effectif de l'IUT 2011-2015 (en nombre d'étudiants)

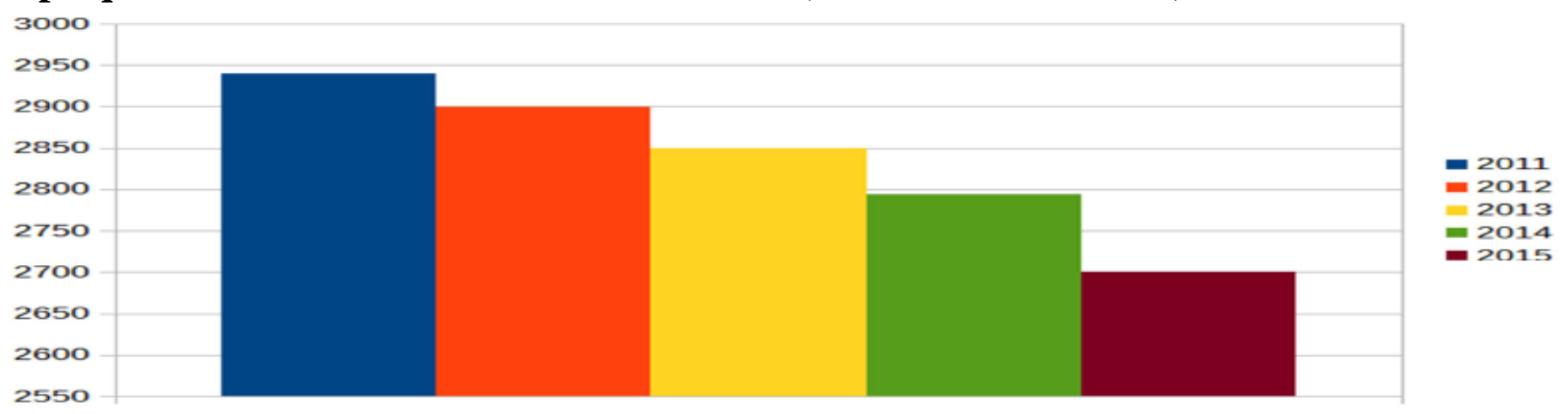

Graphique 4. Évolution des recettes des relations internationales (en €)

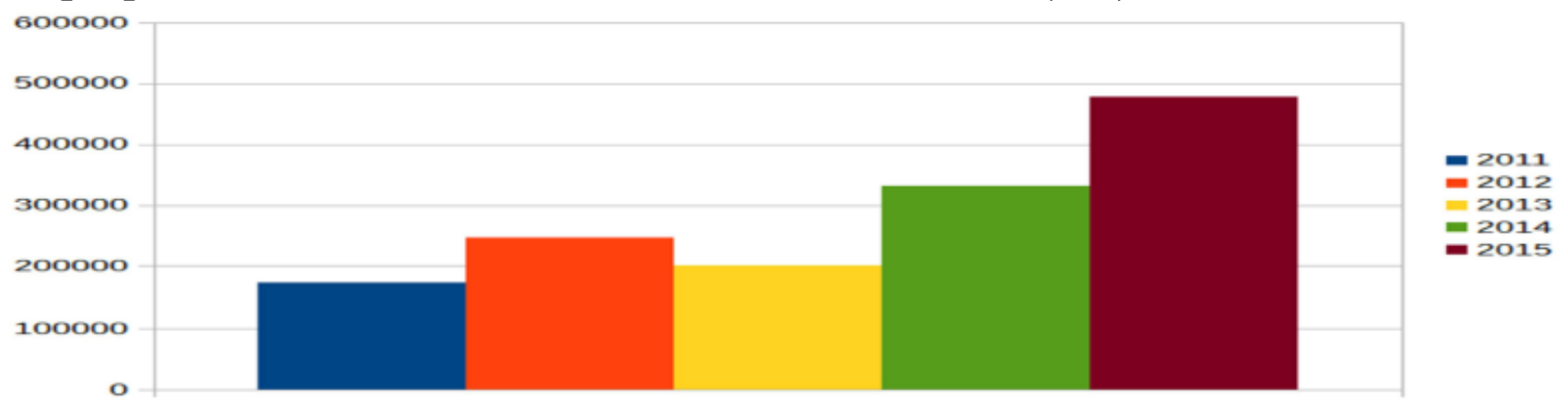

\subsection{Le niveau ministériel}

Un deuxième niveau d'objectifs et d'indicateurs de performance est prescrit par le modèle d'attribution des moyens (modèle SYMPA ${ }^{20}$ ) défini par la DGESIP ${ }^{21}$. Ce modèle est fondé sur deux types d'indicateurs (indicateur d'activité et indicateur de performance) afin de calculer la dotation annuelle de moyens attribuée à chacune des universités françaises. Ces indicateurs portent, d'une part, sur la formation et, d'autre part, sur la recherche. Les indicateurs développés par ce modèle depuis 2009 (tableau 3) et les changements d'indicateurs intervenus pour la construction des budgets des universités pour 2015 (tableau 4) constituent des frictions institutionnelles fortes dans la mesure où les nouveaux indicateurs ou leurs nouveaux modes de calcul impactent les choix budgétaires des universités.

\footnotetext{
${ }^{20}$ Remplacé par le modèle Modal en 2016

${ }^{21}$ Direction Générale de l'Enseignement Supérieur et de l'Insertion Professionnelle
} 
Tableau 3. Évolution indicateurs de formation Sympa - année 2009 (Source : DGESIP)

\begin{tabular}{|c|c|}
\hline & $\begin{array}{l}\text { Indicateurs } \\
\text { FORMATION }\end{array}$ \\
\hline \multirow{3}{*}{ indicateurs d'activité } & $\begin{array}{l}\text { nombre d'étudiants inscrits présents aux examens pondérés } \\
\text { par classe de formation, pour les niveaux L et M }\end{array}$ \\
\hline & enveloppe spécifique « plan licence », nombre d'étudiants en L \\
\hline & enveloppe spécifique « compensation TD = TP » \\
\hline \multirow{2}{*}{$\begin{array}{l}\text { indicateurs d'incitation (ou } \\
\text { de performance) }\end{array}$} & nombre de diplômés de M \\
\hline & valeur ajoutée de la réussite en L et DUT \\
\hline \multicolumn{2}{|r|}{$\begin{array}{l}\text { Indicateurs } \\
\text { RECHERCHE }\end{array}$} \\
\hline indicateur d'activité & nombre d'enseignants-chercheurs «produisants » \\
\hline \multirow{2}{*}{$\begin{array}{l}\text { indicateurs d'incitation (ou } \\
\text { de performance) }\end{array}$} & cotation des laboratoires \\
\hline & nombre de thèses soutenues \\
\hline
\end{tabular}

Tableau 4. Évolution indicateurs de recherche Sympa - année 2015 (Source : DGESIP)

\begin{tabular}{|c|c|}
\hline \multicolumn{2}{|r|}{$\begin{array}{l}\text { Indicateurs } \\
\text { FORMATION }\end{array}$} \\
\hline indicateur d'activité & $\begin{array}{l}\begin{array}{l}\text { Nombre d'étudiants présents aux examens pondérés par } \\
\text { classe de formation }\end{array} \\
\end{array}$ \\
\hline \multirow{5}{*}{$\begin{array}{l}\text { indicateurs d'incitation (ou } \\
\text { de performance) }\end{array}$} & nombre de diplômés de $\mathrm{L}$ et $\mathrm{M}$ \\
\hline & valeur ajoutée de la réussite en L, LP, DUT et M \\
\hline & taux d'étudiants en apprentissage \\
\hline & nombre d'heures stagiaires par enseignants \\
\hline & $\begin{array}{l}\text { taux d'inscrits titulaires d'un bac techno ou pro en 1re année } \\
\text { de DUT }\end{array}$ \\
\hline \multicolumn{2}{|r|}{$\begin{array}{l}\text { Indicateurs } \\
\text { RECHERCHE }\end{array}$} \\
\hline indicateur d'activité & le nombre d'enseignants-chercheurs environnés \\
\hline \multirow{3}{*}{$\begin{array}{l}\text { indicateurs d'incitation (ou } \\
\text { de performance) }\end{array}$} & $\begin{array}{l}\text { nombre de thèses de doctorat soutenues (dont le nombre de } \\
\text { thèses d'exercice en santé pondéré à } 1 / 2 \text { ) }\end{array}$ \\
\hline & $\begin{array}{l}\text { pourcentage d'enseignants-chercheurs membres actifs de } \\
\text { l'IUF }\end{array}$ \\
\hline & $\begin{array}{l}\text { Montant des contributions par enseignant-chercheur des } \\
\text { projets déposés et évalués du 7e PCRDT }\end{array}$ \\
\hline
\end{tabular}

Ainsi, en matière de formation, de nouveaux indicateurs portant sur le taux d'étudiants en apprentissage et sur l'origine des étudiants inscrits en IUT (tableau 4) impactent, par les changements qu'ils provoquent, les décisions budgétaires de l'établissement étudié. Ces changements expliquent l'apparition, à partir de 2015, de ponctuations à la hausse des prévisions de ressources propres (graphique 5) provoquées notamment par un développement des formations en apprentissage. Ils justifient également la réduction des effectifs de cet IUT (graphique 3) imposée par les nouveaux objectifs d'accueil des étudiants issus des filières technologiques.

De même, en matière de recherche, la suppression du concept de «chercheur produisant » (tableau 3) et l'apparition d'un indicateur évaluant le nombre de contrats de recherche financés par l'IUF ${ }^{22}$ ou le PCRDT $^{23}$ (tableau 4) expliquent, à partir de 2015, la réduction des moyens de fonctionnement attribués pour la recherche par l'Université (graphique 1) et l'augmentation des ressources propres (graphique 5).

Graphique 5. Evolution des ressources propres (période 2011-2015)

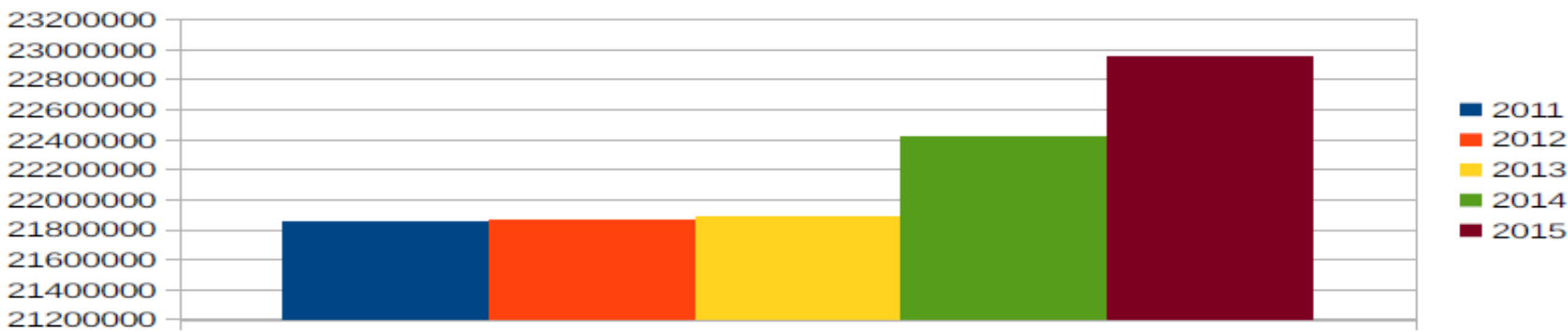




\subsection{Le niveau local}

Un troisième niveau d'apparition d'objectifs et indicateurs de performance se situe au niveau des tableaux de bord et de pilotage développés dans les universités et inspirés par les contrats quinquennaux entre l'État et les universités (tableau 5). Ce niveau révèle les choix de variables qui constituent, dans le modèle de la PET, des frictions cognitives en mesure d'expliquer les ponctuations apparaissant dans les évolutions du budget de l'établissement.

\section{Tableau 5. Indicateurs du tableau de bord de l'université (Source : Université)}

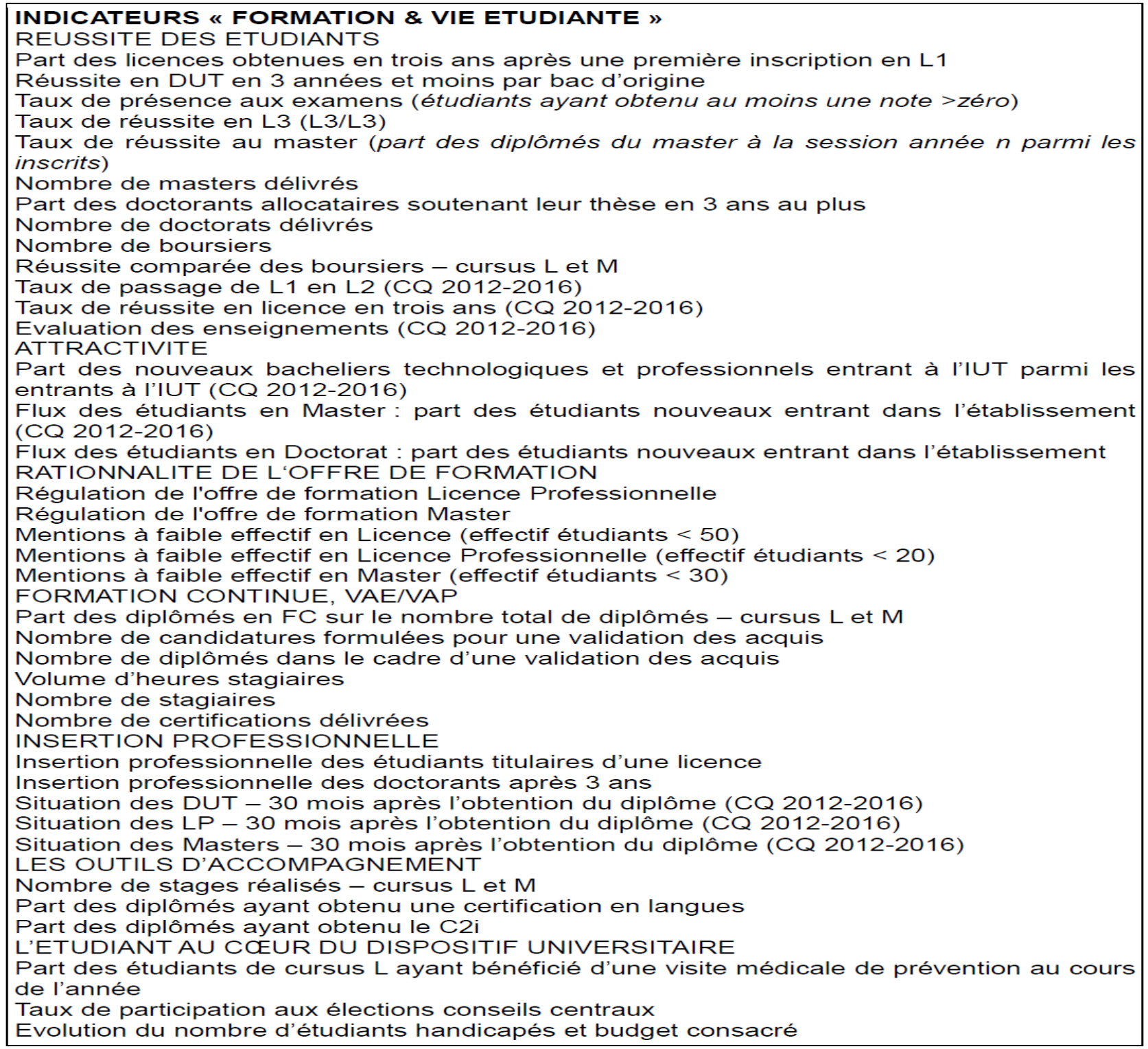

\section{INDICATEURS « PILOTAGE DES MOYENS »}

QUALIFICATION DU DISPOSITIF D'AUTOEVALUATION

Mise en œuvre des procédures par secteur (Scolarité, RH, Finances...)

Implication de la composante dans le développement du processus de contrôle interne

Contribution aux groupes de travail dédiés à la construction des "fiches de procédure " GESTION DES RESSOURCES HUMAINES

Endorecrutement des enseignants chercheurs - MCF et PU

Temps de travail des personnels (annuel)

Optimisation et mutualisation des moyens humains (CQ 2012-2016)

Redéploiements de postes

Taux de demandes de formation (CQ 2012-2016)

Taux effectif de formation (CQ 2012-2016)

Maîtrise de l'évolution des heures complémentaires (CQ 2012-2016)

Maîtrise masse salariale sur ressources d'établissement (CQ 2012-2016)

GESTION DES MOYENS

Evolution et part des ressources générées

Taux d'exécution budgétaire global

Taux d'exécution budgétaire par secteur

Volume des DBM (qualité des prévisions budgétaires)

Taux d'occupation des locaux (CQ 2012-2016)

Recettes de la formation continue (CQ 2012-2016) 
Dans le cadre de notre étude de cas, les changements d'indicateurs intervenus lors de la négociation du contrat quinquennal 2012-2016 imposent à l'établissement étudié de retenir de nouvelles variables de pilotage de la performance justifiant certains ajustements budgétaires. Ainsi, les nouveaux indicateurs portant sur la réussite des étudiants et sur la sélection de ceux provenant de filières technologiques (CQ 2012-2016, tableau 5) représentent des frictions cognitives en mesure d'expliquer la réduction des effectifs de l'IUT constatée au cours de la période 2012-2015 (graphique 3).

De même, l'apparition dans le tableau de bord de l'Université de nouveaux indicateurs portant sur l'insertion professionnelle des étudiants à 30 mois, sur le taux effectif de formation ou sur les nouvelles demandes de formation (CQ 2012-2016) justifient la survenance de ponctuations, notamment à partir de 2013, réduisant le nombre des formations proposées par l'établissement (graphique 6).

\section{Graphique 6. Evolution du nombre de formations (en nombre de formations)}

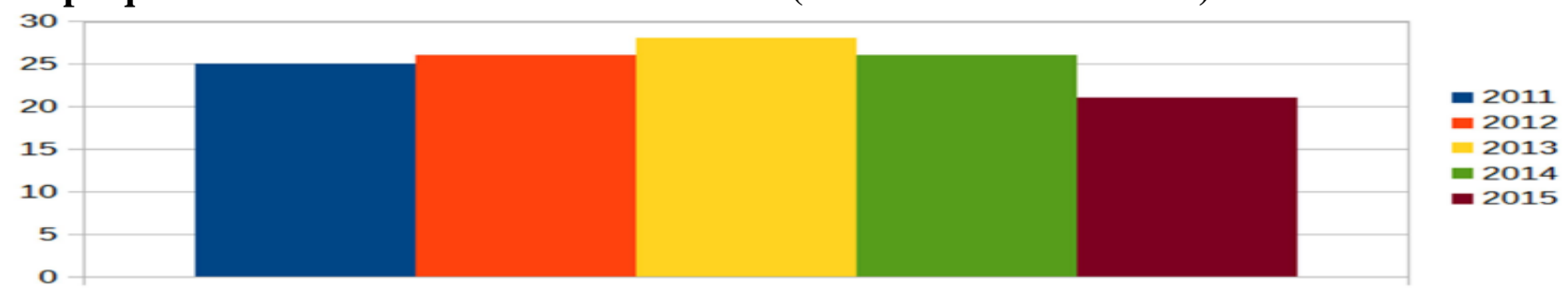

Dans le même ordre d'idée, le contrat quinquennal (CQ 2012-2016) a incité l'Université à faire apparaître dans ses tableaux de bord des indicateurs de gestion de la masse salariale (Maîtrise de l'évolution des heures complémentaires; Maîtrise masse salariale sur ressources d'établissement; Optimisation et mutualisation des moyens humains). Dans le budget de l'IUT étudié, ces changements se répercutent, à partir de 2013, par une stabilisation de la masse salariale (graphique 7), et, à partir de 2012, par de fortes ponctuations à la hausse des heures de vacation (graphique 8) et à la baisse des heures complémentaires (graphique 9).

\section{Graphique 7. Évolution de la Masse salariale P1 (en €)}

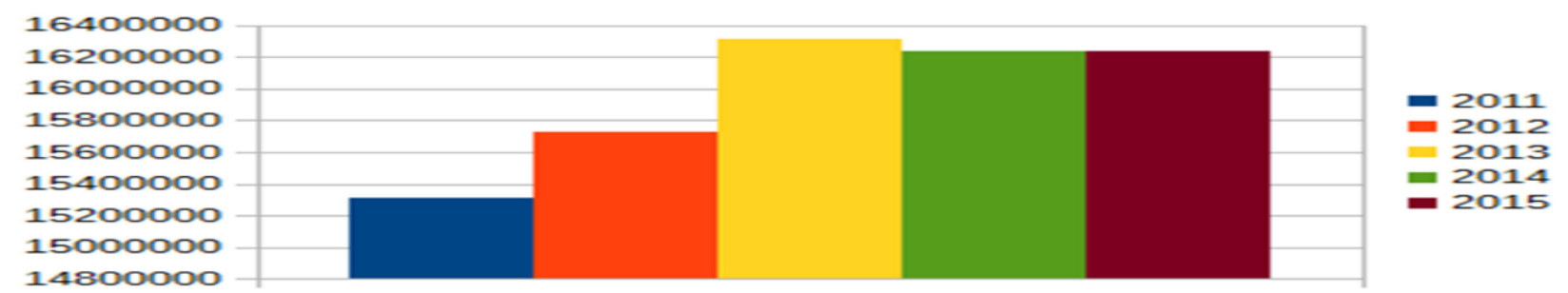

\section{Graphique 8. Evolution du budget des heures de vacations P2 (en €)}

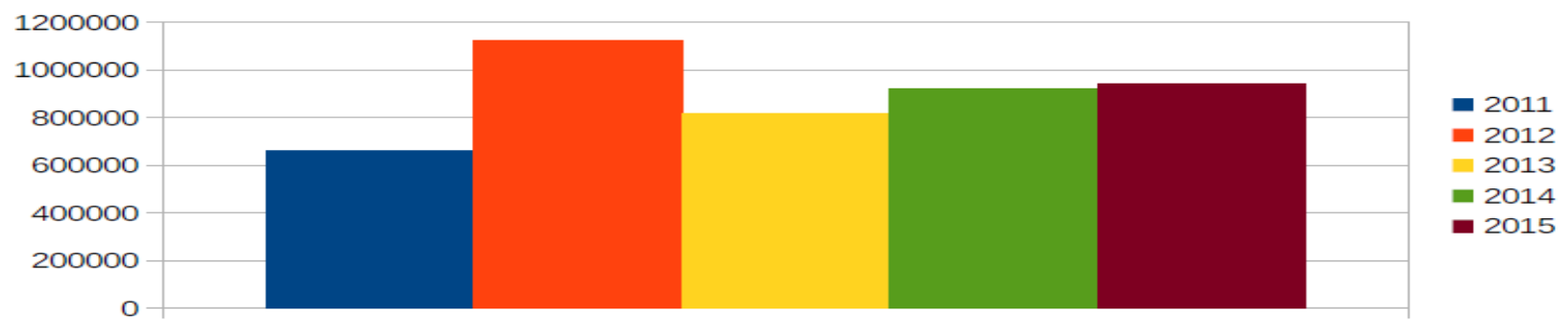

Graphique 9. Evolution du budget des heures complémentaires (en €)

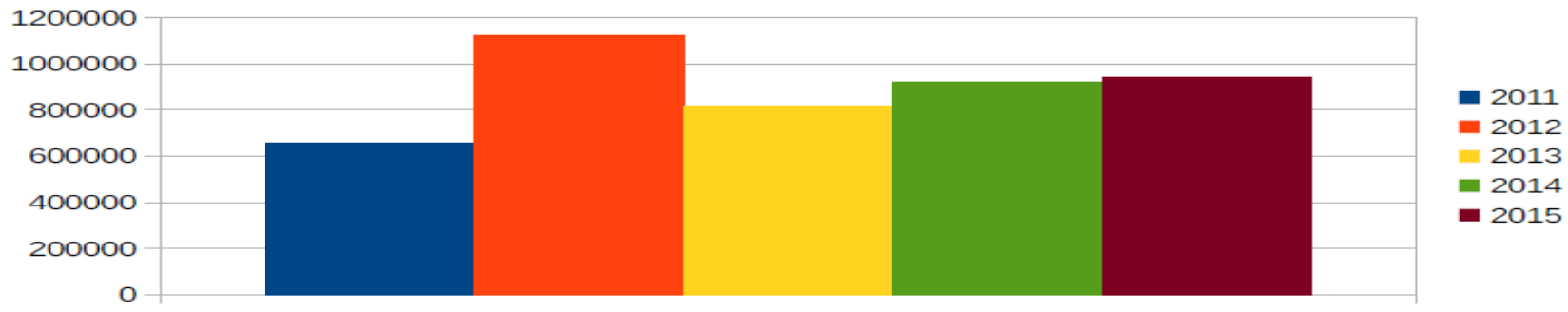

Enfin l'apparition de nouveaux indicateurs de gestion des moyens dans les tableaux de bord de l'Université symbolise la volonté de cette dernière de sélectionner des variables de gestion en accord avec le contrat 
quinquennal. Tel est le cas du taux d'occupation des locaux et du montant des recettes de formation continue (CQ 2012/2016, tableau 5) qui se concrétisent à la fois par une hausse des dotations de fonctionnement, à partir de 2012 (graphique 2), et par une forte ponctuation à la hausse des recettes de formation de continue en 2012 (graphique 10).

\section{Graphique 10. Evolution des recettes de formation continue}

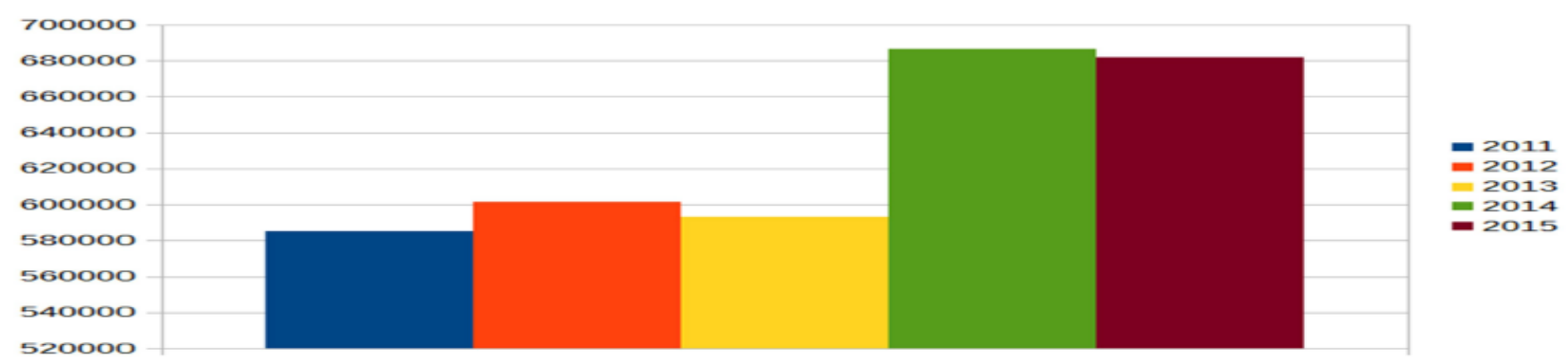

\section{Le processus d'hybridation instrumentale}

Baumgartner et Jones (1993) considèrent que les ponctuations budgétaires s'expliquent par des changements intervenants dans la sélection des problèmes et des variables à traiter par les décideurs publics. Nous démontrons que les changements intervenus dans la définition des indicateurs de performance universitaire, aux niveaux législatif et ministériel (frictions institutionnelles) et au niveau local (frictions cognitives), expliquent les ponctuations budgétaires observées dans l'IUT étudié. Ces ponctuations budgétaires provoquent des frictions instrumentales (figure 1) qui correspondent à une sélection d'indicateurs de performance privilégiés par les décideurs budgétaires en fonction des moyens qu'ils peuvent leur allouer.

Ces trois formes de frictions permettent de décrire le processus d'hybridation entre budget et instruments de mesure de la performance. En effet, au lieu de s'opposer ou de se superposer aux indicateurs de performance, le budget s'articule avec eux au moins à trois niveaux.

Les trois dimensions d'un instrument de gestion décrites par Hatchuel et Weil (1992) permettent d'illustrer comment dans la pratique ces articulations s'opèrent. Pour ces auteurs, les instruments de gestion influencent leurs utilisateurs à la fois par leur philosophie gestionnaire, leur vision simplifiée des relations organisationnelles et leur substrat technique (ibid.). Dans notre étude de cas, nous constatons que les outils de gestion s'influencent également entre eux par l'intermédiaire de ces trois dimensions mises en tension par les trois formes de frictions observées.

Ainsi, nous montrons que les frictions institutionnelles correspondent à des évolutions d'objectifs ou d'indicateurs de performance prescrits aux niveaux législatif et ministériel. Elles permettent d'articuler les indicateurs de performance universitaire et le budget des établissements. Nous qualifions ces articulations de stratégiques car elles attribuent au budget une fonction de pilotage stratégique de la performance à travers la prise en compte des changements d'indicateurs intervenus dans les programmes de performance ministériels. Ces articulations stratégiques s'opèrent par l'hybridation de la logique de résultat des instruments de pilotage de la performance et celle de moyen portée par le budget (figure 1). Le rapprochement entre ces deux logiques modifie la philosophie gestionnaire (ibid.) du budget universitaire qui, dès lors, va avoir, à la fois, un rôle de pilotage des moyens mais aussi de la performance associée à ces moyens (figure 1).

Les frictions cognitives correspondent à l'adaptation, au niveau local, des changements d'indicateurs prescrits aux niveaux supérieurs (figure 1). Ces changements d'indicateurs dans les tableaux de bord provoquent des ponctuations dans les budgets afin de permettre à ces derniers de s'adapter aux évolutions décidées au niveau local. Ces frictions permettent d'articuler les tableaux de bord des universités et les budgets des établissements. Nous qualifions ces articulations d'organisationnelles car elles rapprochent la vision processuelle des organisations définie dans les objectifs transversaux de performance des tableaux de bord des universités (réussite des étudiants, insertion professionnelle, attractivité internationale, etc.) avec celle plus fonctionnelle prise en compte dans les budgets pour attribuer des moyens aux établissements (masse salariale, dépenses de recherche, de formation, etc.).

Les frictions instrumentales, non encore observées dans le modèle de la PET et que nous illustrons dans notre étude de cas, correspondent à une troisième forme d'articulations. Elles sont des frictions au sens de 
Baumgartner et al. (2009) dans la mesure où elles sont le résultat d'une évolution des indicateurs de performance observable dans les ponctuations budgétaires (graphiques 1 à 10). Il est possible de rattacher à chaque ponctuation budgétaire un indicateur de performance ou de ressources (figure 1). Nous qualifions ces articulations d'artéfactuelles car elles modifient le substrat technique du budget qui, par son hybridation avec les instruments de pilotage de la performance, permet maintenant d'associer ces deux types d'indicateurs.

Ces trois formes d'articulations entre le budget et les indicateurs de performance universitaire décrivent un processus d'hybridation instrumentale qui, dans la pratique, fait du budget le principal voire l'unique instrument de pilotage de la performance de l'établissement universitaire étudié. Ceci constitue un résultat important car il met en évidence la capacité des instruments de gestion à se connecter entre eux afin de gérer et simplifier la gestion publique, notamment celle de la performance, qui s'est largement complexifiée avec l'apparition régulière, et souvent non coordonnée, de nouveaux instruments.

A travers notre approche de l'hybridation nous avons effectué le choix de déterminer s'il pouvait exister une hybridation portant sur l'instrument de gestion à proprement parlé aux cotés des formes d'hybridation déjà illustrées dans la littérature portant plus sur des hybridations de comportements, de valeurs, de logiques ou de pratiques.

Figure 1. Le processus d'hybridation instrumentale des administrations publiques

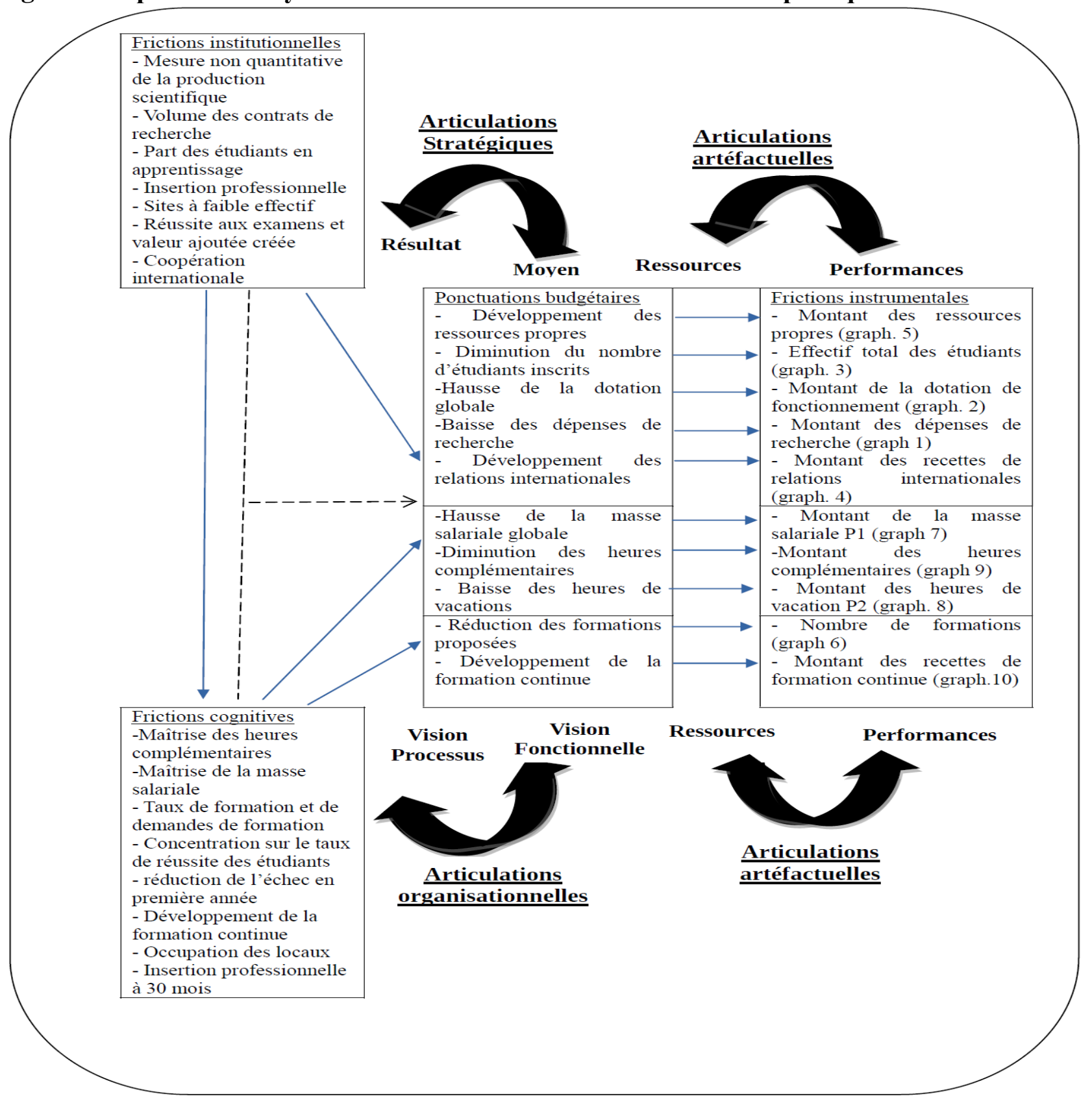




\section{Conclusion}

L'objectif de notre recherche était d'analyser les articulations et combinaisons entre les instruments budgétaires et de pilotage de la performance universitaire à partir d'une analyse des ponctuations budgétaires observées dans l'étude longitudinale du cas d'un IUT.

Plusieurs apports résultent de cette analyse. Le premier d'entre eux est d'ordre heuristique. Il met en évidence l'intérêt d'étudier les données budgétaires des organisations publiques, abondantes et pourtant souvent sous exploitées, afin d'analyser en profondeur les évolutions managériales majeures qui affectent ces organisations depuis une trentaine d'années. Nous avons démontré que la théorie de la PET (Baumgartner et al., 2009) offre des clés de lecture très opérantes pour développer notre compréhension de ces évolutions, notamment instrumentales.

Deux enrichissements théoriques sont ensuite proposés. Un premier propose la description, dans le modèle de la PET, aux côtés de la sélection des problèmes et des variables d'un troisième type de frictions (instrumentales) qui correspond à la sélection d'indicateurs d'évaluation de la performance issue des ponctuations budgétaires observées.

Ce premier apport permet d'illustrer notre deuxième enrichissement théorique représenté par notre proposition de caractérisation du processus d' «hybridation instrumentale » selon trois formes d'articulation (stratégiques, organisationnelles et artéfactuelles). Ce résultat important permet de qualifier de manière plus précise cette forme d'hybridation par les instruments qui est assez peu analysée et pourtant très substantielle notamment dans le secteur public, compte tenu du nombre de nouveaux outils de gestion introduits à partir des années 2000. Ce dernier résultat permet de compléter les résultats obtenus par les travaux portant sur l'appropriation des outils de gestion. En effet, ces deux approches (hybridation et appropriation) présentent un dénominateur commun qui est l'usage des outils intégrant notamment les résistances ou les soutiens des acteurs. Ces deniers ont été bien illustrées dans la littérature par les travaux sur l'appropriation à partir d'une analyse fine des interactions entre les outils et les acteurs. Nous démontrons que l'hybridation instrumentale, en se positionnant à un niveau plus macro-analytique, correspond à un processus d'interactions des instruments de gestion dont l'analyse peut alimenter la compréhension des usages d'un outil spécifique.

En termes de recommandations managériales, notre travail suggère aux managers, dans la construction de leurs instruments de gestion, de prendre en compte leurs articulations avec les instruments déjà en place (notamment les budgets). L'analyse de ces articulations à un niveau stratégique, organisationnel et artéfactuel doit permettre de faciliter l'implantation et l'appropriation de ces nouveaux outils (de Vaujany et Grimand, op.cit.). De plus, l'originalité de notre contribution réside dans la description de l'hybridation des administrations publiques par les instruments. L'analyse de l'instrument, tel que nous l'avons défini, permet de restituer une forme d'hybridation objective. En effet, dans la pratique, les formes d'hybridation publique sont particulièrement apparentes dans les transformations des instruments de gestion. Même si les autres formes d'hybridation (valeurs, logiques, pratiques, usages) sont essentielles dans la compréhension des changements intervenant dans les organisations publiques, il parait également important de décrire comme ces changements s'expriment en terme instrumental. Aussi l'étude de ces différentes hybridations se complètent et se renforcent afin de saisir les transformations publiques actuelles. On ne saurait dire si l'une de ces hybridations précède les autres ou leurs succède. Par contre, elles permettent d'avoir une vision plus large des hybridations dans la sphère publique, ce qui doit inciter les managers publics à élargir leurs réflexions sur l'articulation des instruments de gestion qu'ils construisent.

Néanmoins, ce travail comporte certaines limites. Sur le plan théorique, il sera important dans l'avenir que nous puissions préciser, dans de nouvelles études, comment, de manière souvent récursive, les différentes formes d'hybridation se complètent afin de mieux comprendre, autour d'un ensemble d'instruments de gestion, les changements de comportement, de stratégie ou de relations entre les parties prenantes.

Sur le plan méthodologique, le processus d'hybridation instrumentale que nous avons essayé de définir est probablement plus apparent dans un IUT que dans les autres composantes universitaires, 
pour certaines, plus récemment concerné par l'art. 713.9 (IAE ${ }^{24}$ par exemple) et dans lesquelles les négociations ont été toujours réalisées au sein des universités. Ceci constitue une limite importante de notre travail qui, sur le plan théorique, empêche sans doute la généralisation de nos résultats à l'ensemble des composantes universitaires. Néanmoins, les frictions et les hybridations instrumentales que nous mettons en évidence ici, même si elles ne s'expriment pas avec la même intensité selon les composantes peuvent représenter une grille de lecture pertinente dans d'autres composantes des universités (UFR ${ }^{25}$, IAE) qui avec la LRU ont, à la différence des IUT, accédé à une capacité de négociation beaucoup plus grande dont les directeurs et doyens se sont largement emparés. Cette limite imposera, dans l'avenir, de notamment élargir le spectre scientifique de ce processus d'hybridation. En effet, ce processus dépend d'éléments historiques et contextuels qu'il est difficile d'appréhender (comment l'histoire d'une université et ses formes de gouvernance impactent les formes d'hybridation des instruments). Il sera important de l'analyser dans des composantes présentant des histoires et des modalités de gestion différentes.

\section{Bibliographie}

ABO T. (1994), Hybrid factory: The japanese production system in the United States, Oxford, Oxford Universitiy Press

AGULHON, C. (2018) «Les IUT: de l'autonomie à la subordination ? », Cahiers de la recherche sur l'éducation et les savoirs, Hors-série nº 6, p. 71-89.

AMSLEM T. (2013), Le rôle des systèmes de mesure de la performance dans la gestion des conflits identitaires: le cas de l'entreprise d'insertion ARES, Doctoral dissertation, Paris 1, IAE.

ARNABOLDI M., LAPSLEY I., STECCOLINI, I. (2015), «Performance management in the public sector: The ultimate challenge, Financial Accountability \& Management Vol. 31, n 1, p. 1-22.

BANKER R. D., POTTER G., SRINIVASAN D. (2000), «An empirical investigation of an incentive plan that includes nonfinancial performance measures », The accounting review, Vol. 75, ${ }^{\circ} 1$, p. 65-92.

BAUMGARTNER F.R, JONES, B.D. (1993), Agendas and Instability in American Politics, Chicago, University of Chicago Press.

BAUMGARTNER F.R., BREUNIG C., GREEN-PEDERSEN C., JONES B.D., MORTENSEN P.B., NEYTEMANS M., WALGRAVE S. (2009), «Punctuated Equilibrium in Comparative Perspective », American Journal of Political Science, Vol. 53, n³, p. 602-619

BAUMGARTNER F. R., FOUCAULT M., FRANÇOIS A. (2011) « Chapitre 6/L'incrémentalisme et les ponctuations budgétaires en France». Académique, p. 299-322.

BERRY W. (1990). " The confusing case of budgetary incrementalism : Too many meanings fo a single concept », Journal of Politics, Vol. 52, n 1 , p. 167-196

BOITIER M., RIVIÈRE A. (2013), «Are French universities under control? » Public Money and management, Vol. 33, n², p. 105-110.

BOITIER M., RIVIERE A. (2016) «Les systèmes de contrôle de gestion, vecteurs d'une logique gestionnaire: changement institutionnel et conflits de logiques à l'université », Comptabilité-ContrôleAudit, Vol. 22, n³, p. 47-79.

BOLLECKER M. (2013) « Pourquoi le contrôle de gestion à l'Université est-il un échec ? Politiques et Management Public, Vol. 30, n², p. 221-239

BLAIS, A., KIM, J., FOUCAULT, M. (2010). "Public spending, public deficits and government coalitions." Political Studies, Vol. 58, n5, p. 829-846.

BOURGUIGNON, A. (2006), Les instruments de gestion au risque de la réification, Mémoire d'Habilitation à Diriger des Recherches, Paris: Université Paris-Dauphine.

BOYER R. (1998), «Hybridation et modèle productif : géographie, histoire et théorie » in Actes de GERIPSA : Pourquoi les modèles productifs voyagent ? CCFFA, n²4, décembre, p.7-50.

BUFFAT, A. (2014), «C'est l'étiquette État, mais comme une PME! : Hybridation organisationnelle, sentiments d'appartenance et stratégies identitaires des collaborateurs d'une caisse publique de chômage en Suisse », Revue Internationale des Sciences Administratives, Vol. 80, n¹, p. 71-89.

\footnotetext{
${ }^{24}$ Institution d'Administration des Entreprises

${ }^{25}$ Unité de Formation et de Recherche
} 
CARASSUS D., FAVOREU C., GARDEY D., MAUREL C. (2014), «Les déterminants de l'adoption locales françaises», Finance Contrôle Stratégie Vol. 17, $\mathrm{n}^{\circ} 1$.

DA, A., MAUREL, C., FAVOREU, C. (2018), «Interrelations entre système de contrôle informel et système formel de contrôle dans une organisation complexe: cas d'une université française », Finance Contrôle Stratégie, Vol.21, $\mathrm{n}^{\circ} 1$.

DAMANPOUR F., SCHNEIDER. M. (2008) «Characteristics of Innovation and Innovation Adoption in Public Organizations: Assessing the Role of Managers », Journal of Public Administration Research and Theory Vol.19, n³, p. 495-522

DAVID A., HATCHUEL A. LAUFER R. (coord.) (2000), Les nouvelles fondations des sciences de gestion, Vuibert, collection FNEGE

DENIS J-L., FERLIE E., VAN GESTEL N. (2015), « Understanding Hybridity in Public Organizations », Public Administration, vol. 93, ${ }^{\circ}$ 2, p. 273-289.

DERUJINSKY-LAGUECIR, A., KERN, A., LORINO, P. (2011) «Une approche instrumentale des indicateurs de performance », Management \& Avenir, n`2, p. 111-132.

DREVETON B., LANDE E., PORTAL M. (2012). «Construire un outil de comptabilité de gestion au sein d'une université. Retour sur un acte manqué ». Management et Avenir, $n^{\circ} 54$, p. 126-144

DE VAUJANY, F. X. (2006), «Pour une théorie de l'appropriation des outils de gestion: vers un dépassement de l'opposition conception-usage », Management et Avenir, $n^{\circ} 3$, p. 109-126.

DE VAUJANY, F.X., GRIMAND, A. (2005), « Réflexion sur la place de la perspective appropriative au sein des sciences de gestion », In De la conception à l'usage : vers un management de l'appropriation des outils de gestion (Eds, DE VAUJANY, F.X.). Cormelles-le-Royal : EMS.

EMERY, Y. (2012) «La diversité des motivations des employés publics. Recherche exploratoire dans un contexte post-bureaucratique en Suisse » Revue française d'administration publique, ${ }^{\circ}$ 2, p. 491-515.

EMERY, Y. GIAUQUE, D. (2014) «L'univers hybride de l'administration au XXIe siècle. Introduction » Revue Internationale des Sciences Administratives, Vol. 80, n 1, p. 25-34.

EISSLER, R., RUSSELL, A. JONES, B. D. (2016) «The Transformation of Ideas: The Origin and Evolution of Punctuated Equilibrium Theory » In Contemporary Approaches to Public Policy (pp. 95112). Palgrave Macmillan UK.

EZZAMEL M., ROBSON K., STAPLETON P. (2012). «The logics of budgeting: Theorization and practice variation in the educational field », Accounting, Organizations and Society, Vol. 37, $n^{\circ} 5$, p. 281303.

FERREIRA A., OTLEY, D. (2009), « The design and use of performance management systems: An extended framework for analysis », Management accounting research, Vol. 20, n4, p. 263-282.

FRANZESE, R. J. (2002). "Electoral and Partisan Cycles in Economic Policies and Outcomes." Annual Review of Political Science, $n^{\circ} 5$, p. 369-421.

GUENOUN, M., GOUDARZI, K., CHANDON, J. L. (2016) «Construction et validation d'un modèle hybride de mesure de la qualité perçue des services publics (QSP) », Revue Internationale des Sciences Administratives, Vol. 82, ${ }^{\circ} 1$, p. 223-243.

HATCHUEL A., WEIL B. (1992) L'expert et le système. Gestion des savoirs et métamorphose des acteurs dans l'entreprise industrielle, Economica.

HOQUE, Z. (2005) «Linking environmental uncertainty to non-financial performance measures and performance: a research note », The British Accounting Review, Vol. 37, n², p. 471-481.

JONES B. D., BAUMGARTNER F. R. (2012) «From there to here: Punctuated equilibrium to the general punctuation thesis to a theory of government information processing ». Policy Studies Journal, Vol. 40, $n^{\circ} 1$, p. $1-20$.

KIEFFER R. (1996). «La transférabilité des outils de contrôle de gestion du privé vers les collectivités territoriales », in Le maire entrepreneur ?, Le Duff R. et Rigal J.J., (dir.), PUP, Pau, p. 239-247

LACOUETTE FOUGERE C. LASCOUMES, P. (2013), "L'évaluation : un marronnier de l'action gouvernementale ? », Revue française d'administration publique, Vol. 4, n ${ }^{\circ}$ 148, p. 859-875.

LALONDE, J. F. (2013). « La participation observante en sciences de la gestion: plaidoyer pour une utilisation accrue ». Recherches qualitatives, Vol. 32, $\mathrm{n}^{\circ}$ 2, p. 13-32.

LIBBY, T, LINDSAY, R. M. (2010). "Beyond Budgeting or Budgeting Reconsidered? A Survey of 
North-American Budgeting Practice”. Management Accounting Research, Vol. 21, n 1 , p. 56-75

LORINO, P. (2005). «2. Théories des organisations, sens et action: le cheminement historique, du rationalisme à la genèse instrumentale des organisations ». In Entre connaissance et organisation: l'activité collective (pp. 54-71). La Découverte.

MALMI, T., BROWN, D. A. (2008), « Management control systems as a package : Opportunities, challenges and research directions » Management accounting research, Vol. 19, n 4, p. 287-300.

MAZOUZ, B., SPONEM, S., ROUSSEAU, A. (2015), «Le gestionnaire public en question », Revue française de gestion, $\mathrm{n}^{\circ} 5$, p. 89-104.

MEYER R.E., HAMMERSCHMID G. (2006) «Changing institutional logics and executive identities a managerial challenge to public administration in Austria », American Behavioral Scientist, Vol.49, $\mathrm{n}^{\circ}$, $\mathrm{p}$. 1000-1014

MEYER, R. E., EGGER- PEITLER, I.., HÖLLERER, M. A., ET HAMMERSCHMID, G. (2014), « Of bureaucrats and passionate public managers: Institutional logics, executive identities, and public service motivation », Public Administration, Vol. 92, n4, p. 861-885.

MIGNOT-GERARD S. (2012) «Le gouvernement d'une université face aux investissements d'avenir. Entre réactivité et résistances », Politiques et Management Public, Vol. 29, n³, p. 519-539

MODELL S. (2003) «Goals versus institutions: the development of performance measurement in the Swedish university sector », Management Accounting Research, Vol. 14, $n^{\circ} 4$, p. 333-359.

MOLL J., HOQUE Z. (2011). «Budgeting for legitimacy: The case of an Australian university », Accounting Organizations and Society, Vol. 36, n², p. 86-101.

MOYNIHAN D.P. (2006), "Managing for Results in State Government: Evaluating a Decade of Reform », Public Administration Review, Vol. 66, n¹, p. 78-90.

OIRY, E. (2005). «Qualification et compétence: deux sœurs jumelles? », Revue française de gestion, (5), 13-34.

ORLIKOWSKI, W.J. (2006) «Material Knowing: the Scaffolding of Human Knowledge ability», European Journal of Information Systems, Vol. 15, p. 460-466

ORTON, J. D., WEICK, K. E. (1990). "Loosely coupled systems: A reconceptualization.” Academy of management review, Vol. 15, n², p.203-223

PARKER, L. (2011), «University corporatization: Driving redefinition », Critical Perspectives on Accounting, Vol. 22, p. 434-450

PERETZ H. (2004), Les méthodes en sociologie, Paris, La découverte.

RABARDEL P. (2002), «Le langage comme instrument? Eléments pour une théorie instrumentale étendue», in CLOT, Y. (éd.), Avec Vygotski. La Dispute, Paris, pp. 265-290.

ROMANELLI, E., TUSHMAN, M. L. (1994) « Organizational transformation as punctuated equilibrium: An empirical test », Academy of Management journal, Vol. 37, n5, p. 1141-1166.

RONDEAUX, G. (2014), «Quelles dynamiques d'identification organisationnelles lors de processus de modernisation? Analyse d'une administration belge », Revue Internationale des Sciences Administratives, Vol. 80, $\mathrm{n}^{\circ} 1$, p. 113-132.

SANDELIN, M. (2008) «Operation of management control practices as a package-A case study on control system variety in a growth firm context », Management Accounting Research, Vol. 19, n4, p. 324-343.

SOULE, B. (2007). «Observation participante ou participation observante ? Usages et justifications de la notion de participation observante en sciences sociales. » Recherches qualitatives, Vol. 27, $\mathrm{n}^{\circ} 1$, p. $127-140$

SMITH W., LEWIS M. W. (2011). "Toward a theory of paradox: A dynamic equilibrium model of organizing", Academy of Management Review, Vol. 36, n² 2, p. 381-403.

TEELKEN C. (2015), «Hybridity, Coping Mechanism and academic Performance management: Comparing Three Countries », Public Administration, Vol. 93, n 2, p. 307-323.

TROSA S. (1989). «Grandeur et décadence du mimétisme avec le secteur privé », Politiques et Management Public, Vol. 7, ${ }^{\circ}$ 1, p. 237-249

WILDAVSKY, A. (1964), The Politics of the Budgetary Process, Little, Brown. 